\title{
Association between Platelet-to-Lymphocyte Ratio and Contrast-Induced Nephropathy in Patients Undergoing Percutaneous Coronary Intervention for Acute Coronary Syndrome
}

\author{
Muhammed Bora Demircelik ${ }^{a} \quad$ Alparslan Kurtul ${ }^{c}$ Hakan Ocek $^{c}$ \\ Muzaffer Cakmak $^{b}$ Cagın Ureyen ${ }^{d}$ Beyhan Eryonucu ${ }^{a}$ \\ Departments of ${ }^{a}$ Cardiology and ${ }^{b}$ Internal Medicine, Faculty of Medicine, Turgut Ozal \\ University, and ${ }^{\mathrm{C}}$ Department of Cardiology, Ankara Education and Research Hospital, \\ Ankara, and d Department of Cardiology, Antalya Education and Research Hospital, \\ Antalya, Turkey
}

\section{Key Words}

Hematological parameters · Platelet-to-lymphocyte ratio · Contrast-induced nephropathy ·

Acute coronary syndrome

\begin{abstract}
Objective: Contrast-induced nephropathy (CIN) is associated with significantly increased morbidity and mortality after percutaneous coronary intervention (PCI). Patients with acute coronary syndrome (ACS) are at higher risk of CIN. The platelet-to-lymphocyte ratio (PLR) is closely linked to inflammatory conditions. We hypothesized that PLR levels on admission can predict the development of CIN after PCI for ACS. Subjects and Methods: A total of 426 patients (mean age $63.17 \pm 13.01$ years, $61.2 \%$ males) with ACS undergoing PCI were enrolled in this study. Admission PLR levels were measured before PCI. Serum creatinine values were measured before and within $72 \mathrm{~h}$ after the administration of contrast agents. Patients were divided into 2 groups: the CIN group and the no-CIN group. CIN was defined as an increase in serum creatinine level of $\geq 0.5 \mathrm{mg} / \mathrm{dl}$ or $25 \%$ above baseline within $72 \mathrm{~h}$ after contrast administration. Results: CIN developed in 53 patients (15.9\%). Baseline PLR was significantly higher in patients who developed CIN compared to those who did not (160.8 \pm 29.7 and 135.1 \pm 26.1 , respectively; $p<0.001$ ). Multivariate analyses found that PLR [odds ratio (OR) 3.453, $95 \%$ confidence interval (CI) 1.453-8.543; $p=0.004]$ and admission creatinine (OR 6.511, 95\% CI 1.759-11.095; $p=0.002$ ) were independent predictors of CIN. Conclusions: The admission PLR level is an independent predictor of the development of CIN after PCI in ACS.
\end{abstract}




\section{CardioRenal Medicine}

Demircelik et al.: Association between Platelet-to-Lymphocyte Ratio and CIN in Patients Undergoing Percutaneous Coronary Intervention for ACS

\section{Introduction}

Contrast-induced nephropathy (CIN) is a serious complication of invasive cardiovascular procedures. The incidence of CIN is $2 \%$ for the general population. However, patients undergoing percutaneous coronary intervention (PCI) are at greater risk, and patients with diabetes or previous renal impairment have a risk of almost $50 \%[1,2]$.

The development of CIN after PCI is associated with poor clinical outcomes including prolonged hospitalization, increased costs, increased rates of end-stage renal failure, myocardial infarction, repeat revascularization, and short- and long-term mortality [3-6]. Furthermore, patients with acute coronary syndrome (ACS) have a 3-fold higher risk of developing CIN [7, 8]. Because CIN occurs more frequently after urgent PCI in patients with ST-segment elevation myocardial infarction (STEMI) and non-STEMI [9], objective, rapidly available and reliable markers may be useful for the identification of patients at risk of developing CIN.

Platelet-to-lymphocyte ratios (PLRs) have recently been introduced as markers of several inflammatory conditions not only in malignancies but also in other pathologies involving the heart, thyroid, kidney and liver, and even diabetes mellitus, metabolic syndrome, and essential hypertension [10].

In the present study, we investigated whether PLR on admission is an independent risk factor that predicts the development of CIN in patients with ACS with ST-segment elevation (STE-ACS) and unstable angina/non-ST-segment elevation (NSTE-ACS) to undergo interventional therapy.

\section{Subjects and Methods}

\section{Study Population}

Between November 2009 and December 2012, a total of 426 consecutive patients (mean age $63.17 \pm$ 13.01 years, $61.2 \%$ males) were identified with acute STE-ACS or NSTE-ACS undergoing emergency PCI. After an evaluation according to inclusion and exclusion criteria, 333 patients were enrolled in our study (fig. 1).

Patients with STE at the J point in 2 or more consecutive leads (with the cutoff point being $>0.2 \mathrm{mV}$ in leads $\mathrm{V} 1$, V2, or V3, and $>0.1 \mathrm{mV}$ in the other leads) and an elevation of the cardiac troponin T level greater than the upper limit of normal were defined as having STE-ACS [11]. Patients with ST-segment depression, T-wave inversion, or no electrocardiographic abnormalities and/or elevation of the cardiac troponin T level greater than the upper limit of normal were defined as having NSTE-ACS [12]. We excluded patients receiving longterm peritoneal or hemodialysis treatment, or those who underwent a renal transplantation or received administration of metformin, nonsteroidal anti-inflammatory drugs, aminoglycosides, or acetylcysteine 1 week before or after PCI. Patients were also excluded if they had intra-aortic balloon pump support before PCI because of cardiogenic shock, cardiac surgery for coronary revascularization, severe chronic heart failure (New York Heart Association class $\geq 3$ ), and contrast exposure 2 weeks before PCI or died during PCI. Patients with an active infection and a history of collagen vascular disease were excluded from the study. The study protocol was approved by the local ethics committee, and written informed consent was obtained from all participants.

\section{Study Protocol and Laboratory Analysis}

In all patients, antecubital venous blood samples for the laboratory analysis were drawn on admission in the emergency room before angiography. Common blood count parameters stored in citrate-based anticoagulated tubes were measured by the Sysmex K-1000 auto-analyzer (Bohemia, N.Y., USA) within 5 min of sampling. Glucose, creatinine, blood urea nitrogen, lipid profile [total cholesterol, low-density lipoprotein (LDL) and high-density lipoprotein cholesterol], and triglyceride were determined by standard methods. High-sensitivity C-reactive protein (hs-CRP) levels were also measured. Immediately after intervention, all patients underwent hydration with intravenous isotonic saline $(0.9 \%)$ at a rate of $1 \mathrm{ml} / \mathrm{kg} / \mathrm{h}$ for $12 \mathrm{~h}$ (or 0.5 $\mathrm{ml} / \mathrm{kg} / \mathrm{h}$ for $12 \mathrm{~h}$ in cases of overt heart failure, including 5 patients from the CIN group and 3 patients from 


\section{CardioRenal Medicine}

Fig. 1. Diagram of the 436 patients who were enrolled in the study after inclusion and exclusion criteria evaluation.

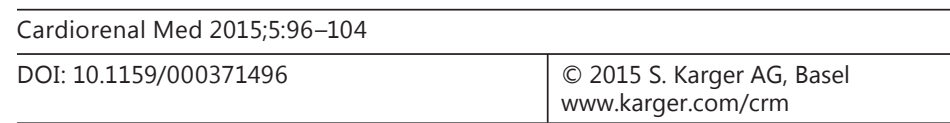

Demircelik et al.: Association between Platelet-to-Lymphocyte Ratio and CIN in

Patients Undergoing Percutaneous Coronary Intervention for ACS

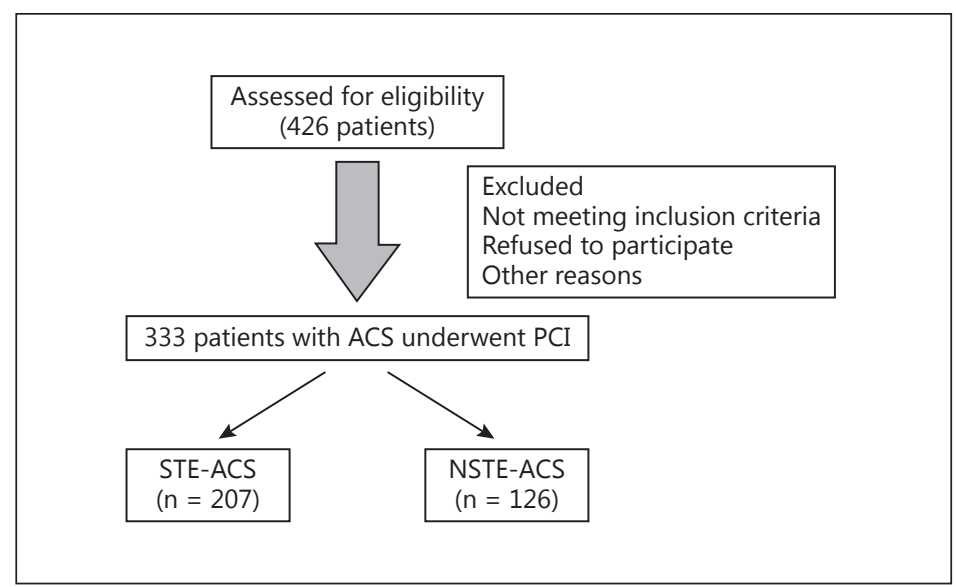

the no-CIN group). Any nephrotoxic medications (i.e., metformin and nonsteroidal anti-inflammatory drugs) were suspended on admission. Serum creatinine was also measured at 24,48 , and $72 \mathrm{~h}$ after contrast medium administration. Patients were divided into 2 groups: the CIN group and the no-CIN group. CIN was defined as an increase in serum creatinine level of $\geq 0.5 \mathrm{mg} / \mathrm{dl}$ or $\geq 25 \%$ above baseline within $72 \mathrm{~h}$ after contrast administration [13]. High-contrast volume was defined as the administration of a contrast volume of $>140$ $\mathrm{ml}$ [4]. The estimated glomerular filtration rate was calculated using the Modification of Diet in Renal Disease (MDRD) equation [5]. Left ventricular ejection fraction (LVEF) was measured using the Simpson method according to the recommendations of the American Society of Echocardiography [14].

\section{Coronary Intervention Procedures and Medications}

Coronary angiography (CA) (Siemens Axiom Artis zee 2008; Siemens Healthcare, Erlangen, Germany) was performed using the femoral approach according to standard clinical practice. On admission, all patients in the emergency department received a bolus of 5,000 units of intravenous unfractionated heparin, followed by additional intraprocedural boluses to maintain an activated clotting time of 200-250 s (>300 s when tirofiban was not used), acetylsalicylic acid (300 mg orally), and a clopidogrel loading dose of $600 \mathrm{mg}$ orally. Nonionic, isoosmolar contrast medium (iodixanol, Visipaque $320 \mathrm{mg} / 100 \mathrm{ml}$; GE Healthcare, Cork, Ireland) was used to visualize the coronary arteries. PCI was performed immediately after diagnostic CA when appropriate.

Coronary stenting was performed using standard techniques. Thrombolysis in myocardial infarction grade 3 coronary flow in the treated vessel with a residual stenosis $<50 \%$ was considered a successful PCI. The use of a glycoprotein IIb/IIIa inhibitor as well as of bare-metal or drug-eluting stents was left to the discretion of the interventional cardiologist. Additional use of thrombectomy was recommended depending on thrombus in the infarct-related artery. After CA, all patients continued to take aspirin (100 mg/day orally) indefinitely and clopidogrel (75 mg/day) for at least 12 months.

\section{Statistical Analysis}

We decided on the sample size of the study by using a program named 'Power and Sample Size Calculator' (Statistical Solutions, Cottage Grove, Wisc., USA; www.statisticalsolutions.net/pss_calc.php). Statistical analysis was performed with the Statistical Package for Social Sciences for Windows, version 18.0 (SPSS Inc., Chicago, Ill., USA). Continuous variables were compared using the Student t test. In case of non-normal distribution, nonparametric methods were used (Mann-Whitney U test). All values are expressed as the mean \pm standard deviation or the median and interquartile range. Models were developed with stepwise techniques and on account of potential confounding factors as well as of variables that are shown to be statistically significant at univariate analysis. The results of this model were presented as odds ratios (ORs) and 95\% confidence intervals (CIs). To identify the independent parameters associated with CIN, multivariable logistic regression analysis was used. According to the results of the univariate analysis, age, smoking, LVEF, hemoglobin, creatinine, PLR, total cholesterol, LDL cholesterol, hs-CRP, uric acid, troponin T, SYNTAX (SYNergy between PCI with TAXus and cardiac surgery) score, the presence of multivessel disease (MVD), and the presence of chronic total occlusion (CTO) were analyzed with the multivariate logistic regression model. The 
Demircelik et al.: Association between Platelet-to-Lymphocyte Ratio and CIN in Patients Undergoing Percutaneous Coronary Intervention for ACS

Table 1. Baseline characteristics of the study patients

\begin{tabular}{lccr}
\hline Variable & $\begin{array}{l}\text { No-CIN group } \\
(\mathrm{n}=280,84.1 \%)\end{array}$ & $\begin{array}{c}\text { CIN group } \\
(\mathrm{n}=53,15.9 \%)\end{array}$ & p value \\
\hline Age, years & $60.2 \pm 12.3$ & $68.2 \pm 12.6$ & $<0.001$ \\
Males & $190(67.8)$ & $35(66.0)$ & 0.765 \\
BMI & $26.83 \pm 3.53$ & $27.65 \pm 5.53$ & 0.445 \\
Systolic blood pressure, mm Hg & $129 \pm 12.1$ & $126 \pm 11.7$ & 0.675 \\
Diastolic blood pressure, mm Hg & $79 \pm 10$ & $77 \pm 12$ & 0.876 \\
Hypertension & $135(48.2)$ & $32(60.3)$ & 0.210 \\
Diabetes mellitus & $105(37.5)$ & $17(32.0)$ & 0.365 \\
Smoking & $120(42.8)$ & $28(52.8)$ & 0.432 \\
Hyperlipidemia & $88(31.4)$ & $20(37.7)$ & 0.345 \\
Prior CABG & $20(7.1)$ & $5(9.4)$ & 0.543 \\
Prior myocardial infarction & $30(10.7)$ & $4(7.5)$ & 0.798 \\
LVEF, \% & $45 \pm 10.0$ & $39 \pm 9$ & $<0.001$ \\
LVEF <40\%, \% & 21.1 & 40 & 0.02 \\
Type of ACS $\quad$ & & 0.698 \\
$\quad$ STE-ACS & $175(62.5)$ & $32(60.3)$ & 0.765 \\
$\quad$ NSTE-ACS & $105(37.5)$ & $21(39.7)$ & \\
$\quad$ ACEI or ARB & 28.3 & 26.1 & 0.756 \\
$\quad$ Statins & 24.4 & 25.4 & 0.876 \\
\hline
\end{tabular}

Figures in parentheses are percentages. ACEI = Angiotensin-converting enzyme inhibitor; $\mathrm{ARB}=$ angiotensin receptor blocker; $\mathrm{BMI}=$ body mass index; $\mathrm{CABG}=$ coronary artery bypass graft.

receiver operating characteristic (ROC) analysis was performed to determine the best cutoff value of PLR, and the sensitivity and specificity at that point were obtained for predicting the development of CIN. $p$ values $<0.05$ were considered statistically significant.

\section{Results}

The study population consisted of 426 patients (mean age $63.17 \pm 13.01$ years, $61.2 \%$ males) who had measurements of baseline PLR and creatinine, followed by additional daily measures of creatinine up to $72 \mathrm{~h}$. A total of 53 patients (15.9\%) developed CIN. The baseline clinical characteristics of the patient population stratified by CIN are summarized in table 1 . Patients in the CIN group were significantly older than those in the no-CIN group (68.2 \pm 12.6 vs. $60.2 \pm 12.3$ years, respectively; $p<0.001$ ) and had a significantly lower proportion of smoking ( $\mathrm{p}<0.001)$ compared with patients in the no-CIN group. LVEF was also lower in the CIN group ( $\mathrm{p}<0.001)$. There were no significant differences between the groups regarding gender, hypertension, diabetes mellitus, hyperlipidemia, prior myocardial infarction, prior stroke, prior medications, in-hospital medications, and type of ACS.

The baseline laboratory measurements of the study patients are shown in table 2. PLR at baseline was significantly higher in patients who developed CIN compared to those who did not $(160.8 \pm 29.7$ and $135.1 \pm 26.1$, respectively; $\mathrm{p}<0.001)$. PLR according to risk of developing CIN are shown in table 2. In addition to having elevated PLR, patients who developed CIN had significantly higher baseline serum creatinine, peak troponin $\mathrm{T}$, and uric acid levels, but significantly lower baseline hemoglobin, total cholesterol, LDL, and estimated glomerular filtration rate than those in whom CIN did not occur. Patients with CIN had a higher preva- 
Demircelik et al.: Association between Platelet-to-Lymphocyte Ratio and CIN in Patients Undergoing Percutaneous Coronary Intervention for ACS

Table 2. Common blood count parameters of patients

\begin{tabular}{|c|c|c|c|}
\hline Variables & CIN group $(n=53)$ & No-CIN group $(n=280)$ & $\mathrm{p}$ value \\
\hline Hemoglobin, g/dl & $13.4 \pm 1.6$ & $13.0 \pm 1.8$ & 0.48 \\
\hline White blood cell count, $\times 10^{3} / \mathrm{mm}^{3}$ & $12.0 \pm 3.5$ & $12.6 \pm 3.9$ & 0.58 \\
\hline Platelet count, $\times 10^{3} / \mathrm{mm}^{3}$ & $248 \pm 70.8$ & $250.3 \pm 66.3$ & 0.82 \\
\hline Hematocrit, \% & $41 \pm 6.6$ & $41 \pm 5.3$ & 0.67 \\
\hline Red blood cell distribution width, $\%$ & $13.0 \pm 2.1$ & $13.5 \pm 1.4$ & 0.62 \\
\hline Red blood cell count, $\times 10^{6} / \mathrm{ml}$ & $4.2 \pm 0.6$ & $4.1 \pm 0.3$ & 0.71 \\
\hline Mean corpuscular volume, fl & $82.0 \pm 5.1$ & $84.0 \pm 5.6$ & 0.59 \\
\hline Mean platelet volume, fl & $8.3 \pm 1.2$ & $8.4 \pm 1.4$ & 0.60 \\
\hline PLR & $160.8 \pm 29.7$ & $135.1 \pm 26.1$ & $<0.001$ \\
\hline Serum creatinine, $\mathrm{mg} / \mathrm{dl}$ & $1.40 \pm 0.37$ & $1.06 \pm 0.26$ & $<0.001$ \\
\hline $\mathrm{eGFR}, \mathrm{ml} / \mathrm{min} / 1.73 \mathrm{~m}^{2}$ & $51.3 \pm 17.4$ & $71.1 \pm 20.1$ & $<0.001$ \\
\hline $\mathrm{HbA}_{1 \mathrm{c}}, \%$ & $7.1 \pm 1.83$ & $7.0 \pm 1.78$ & 0.697 \\
\hline Serum glucose on admission, $\mathrm{mg} / \mathrm{dl}$ & $157 \pm 76$ & $155 \pm 77$ & 0.777 \\
\hline Total cholesterol, mg/dl & $188 \pm 48$ & $167 \pm 55$ & 0.567 \\
\hline Triglyceride, mg/dl & $131 \pm 54$ & $140 \pm 53$ & 0.438 \\
\hline $\mathrm{LDL}, \mathrm{mg} / \mathrm{dl}$ & $123 \pm 44$ & $120 \pm 39$ & 0.543 \\
\hline High-density lipoprotein, mg/dl & $44 \pm 12$ & $41 \pm 11$ & 0.876 \\
\hline hs-CRP, mg/l & $8.44 \pm 1.43$ & $7.08 \pm 0.9$ & 0.02 \\
\hline Peak CK-MB, ng/ml & $50.3(2.55-426)$ & $33.4(0.8-435)$ & 0.199 \\
\hline Peak troponin $\mathrm{T}, \mathrm{ng} / \mathrm{ml}$ & $1,800(44.1-10,000)$ & $1,040(4.8-10,000)$ & 0.003 \\
\hline Serum uric acid, mg/dl & $7.1 \pm 1.87$ & $6.5 \pm 1.73$ & $<0.001$ \\
\hline
\end{tabular}

Figures in parentheses are ranges. $\mathrm{CK}-\mathrm{MB}=$ Creatine kinase myocardial band; $\mathrm{eGFR}=$ estimated glomerular filtration rate; $\mathrm{HbA}_{1 \mathrm{c}}=$ hemoglobin $\mathrm{A}_{1 \mathrm{c}}$.

lence of MVD, presence of CTO in non-culprit vessels, and a higher SYNTAX score as well as a lower stent diameter compared with those without CIN. However, the location of the culprit lesion, the contrast volume, procedure duration, and procedure success rate did not significantly differ between patients who developed CIN and those who did not. Angiographic and procedural characteristics of the study patients are listed in table 3. The ROC curve analysis of PLR for predicting CIN is shown in figure 2. PLR $\geq 148.3$ measured on admission had a $75.6 \%$ sensitivity and $72.7 \%$ specificity in predicting CIN.

The effects of multiple variables were analyzed with univariate and multivariate logistic regression analyses. According to the results of univariate analysis, age, smoking, LVEF, hemoglobin, creatinine, PLR $\geq 148.3$, total cholesterol, LDL cholesterol, hs-CRP, uric acid, troponin T, SYNTAX score, the presence of MVD, and the presence of CTO were analyzed with the multivariate logistic regression model. At multivariate analyses, PLR (OR 3.453, 95\% CI 1.453-8.543; $p=0.004$ ) and admission creatinine (OR 6.511, 95\% CI 1.759-11.095; $p=0.002$ ) were still significant independent predictors of the development of CIN after PCI in patients with ACS (table 4).

\section{Discussion}

To our knowledge, our study is the first to demonstrate that PLR is an important predictor of CIN in patients with ACS. Previous studies indicated that a higher PLR value emerged as a significant independent predictor of long-term survival in patients who presented with nonSTEMI [12]. 
Demircelik et al.: Association between Platelet-to-Lymphocyte Ratio and CIN in Patients Undergoing Percutaneous Coronary Intervention for ACS

Table 3. Angiographic and procedural characteristics and medications administered to patients

\begin{tabular}{|c|c|c|c|}
\hline Variable & No-CIN group $(\mathrm{n}=280)$ & CIN group $(\mathrm{n}=53)$ & $\mathrm{p}$ value \\
\hline Total time of procedure, min & $36.56 \pm 17.01$ & $39.08 \pm 13.13$ & 0.153 \\
\hline Total amount of contrast, ml & $160 \pm 15$ & $168 \pm 19$ & 0.234 \\
\hline High-contrast volume & $168(60)$ & $33(62.2)$ & 0.546 \\
\hline MVD & $143(51.1)$ & $39(73.5)$ & 0.003 \\
\hline СТO & $45(16.1)$ & $19(35.8)$ & 0.007 \\
\hline SYNTAX score & $14.34 \pm 8.23$ & $19 \pm 12.46$ & $<0.001$ \\
\hline \multicolumn{4}{|l|}{ Culprit vessel } \\
\hline Left main coronary artery & $1(0.4)$ & $0(0)$ & 0.342 \\
\hline Left anterior descending artery & $135(48.2)$ & $25(47.1)$ & \\
\hline Left circumflex artery & $55(19.6)$ & $12(22.6)$ & \\
\hline Right coronary artery & $76(27.1)$ & $15(28.3)$ & \\
\hline Saphenous vein graft stent implantation & $13(4.6)$ & $1(1.9)$ & \\
\hline Door-balloon time, $\min$ & $108 \pm 28$ & $106 \pm 33$ & 0.688 \\
\hline Stent implantation & $260(92.8)$ & $45(84.9)$ & 0.01 \\
\hline Total length of stent, $\mathrm{mm}$ & $23.11 \pm 10.87$ & $24.18 \pm 11.43$ & 0.565 \\
\hline Stent diameter, $\mathrm{mm}$ & $3.21 \pm 0.41$ & $3.13 \pm 0.54$ & 0.124 \\
\hline Procedural success & $220(78.5)$ & $36(67.9)$ & 0.07 \\
\hline \multicolumn{4}{|l|}{ In-hospital medications, $\%$} \\
\hline Statin & 73.3 & 70.8 & 0.243 \\
\hline ACEI or ARB & 72.6 & 65.9 & 0.645 \\
\hline Tirofiban & 36.3 & 28.1 & 0.128 \\
\hline$\beta$-Blocker & 89.1 & 78.2 & 0.06 \\
\hline Diuretic & 22.8 & 26.1 & 0.778 \\
\hline Clopidogrel & 97.6 & 95.1 & 0.189 \\
\hline
\end{tabular}

Figures in parentheses are percentages. ACEI = Angiotensin-converting enzyme inhibitor; ARB = angiotensin receptor blocker.

Table 4. Univariate and multivariate logistic regression analysis of the association between CIN and multiple parameters

\begin{tabular}{lcccc}
\hline Variables & Univariate analysis & p value & Multivariate analysis & $p$ value \\
\hline Age & $1.170(1.053-1.195)$ & $<0.001$ & $1.023(0.980-1.156)$ & 0.325 \\
Smoking & $0.220(0.114-0.425)$ & $<0.001$ & $0.372(0.150-1.231)$ & 0.117 \\
PLR & $19.432(11.042-27.421)$ & $<0.001$ & $3.453(1.435-8.543)$ & 0.004 \\
LVEF & $0.897(0.804-0.927)$ & $<0.001$ & $0.72(0.934-1.013)$ & 0.244 \\
Hemoglobin & $0.711(0.604-0.776)$ & $<0.001$ & $0.968(0.924-1.011)$ & 0.165 \\
Creatinine & $13.563(6.104-21.421)$ & $<0.001$ & $6.511(1.759-11.095)$ & 0.002 \\
Uric acid & $1.554(1.1224-1.627)$ & $<0.001$ & $1.152(1.260-1.486)$ & 0.345 \\
hs-CRP & $1.111(1.098-1.327)$ & $<0.001$ & $1.125(0.895-1.414)$ & 0.176 \\
Troponin T & $1.226(1.180-1.358)$ & 0.002 & $1.033(0.932-1.146)$ & 0.132 \\
Total cholesterol (mg/dl) & $0.991(0.904-0.998)$ & 0.030 & $1.122(0.092-1.236)$ & 0.145 \\
LDL (mg/dl) & $0.888(0.814-0.929)$ & 0.012 & $1.093(0.995-1.140)$ & 0.178 \\
SYNTAX score & $1.127(1.104-1.328)$ & $<0.001$ & $1.111(0.981-1.281)$ & 0.213 \\
MVD & $2.281(2.004-4.110)$ & 0.003 & $0.563(0.359-1.547)$ & 0.111 \\
CTO & $2.108(1.994-2.429)$ & 0.008 & $1.151(0.562-3.613)$ & 0.129 \\
\hline
\end{tabular}

Data are given as ORs with 95\% CIs in parentheses. CK-MB = Creatine kinase myocardial band; eGFR = estimated glomerular filtration rate; $\mathrm{HbA}_{1 \mathrm{c}}=$ hemoglobin $\mathrm{A}_{1 \mathrm{c}}$. 


\section{CardioRenal Medicine}

Fig. 2. PLR ROC curve for the development of CIN. The CIN ROC curve was plotted to verify the optimum cutoff point for PLR, which was 148.3. The AUC for PLR was 71.4\% (95\% CI 0.665-0.764), with a sensitivity of $75.6 \%$ and a specificity of $72.2 \%$.

\begin{tabular}{l|l}
\hline Cardiorenal Med 2015;5:96-104 \\
\hline DOI: 10.1159/000371496 & $\begin{array}{l}\text { @ 2015 S. Karger AG, Basel } \\
\text { www.karger.com/crm }\end{array}$ \\
\hline
\end{tabular}

Demircelik et al.: Association between Platelet-to-Lymphocyte Ratio and CIN in Patients Undergoing Percutaneous Coronary Intervention for ACS

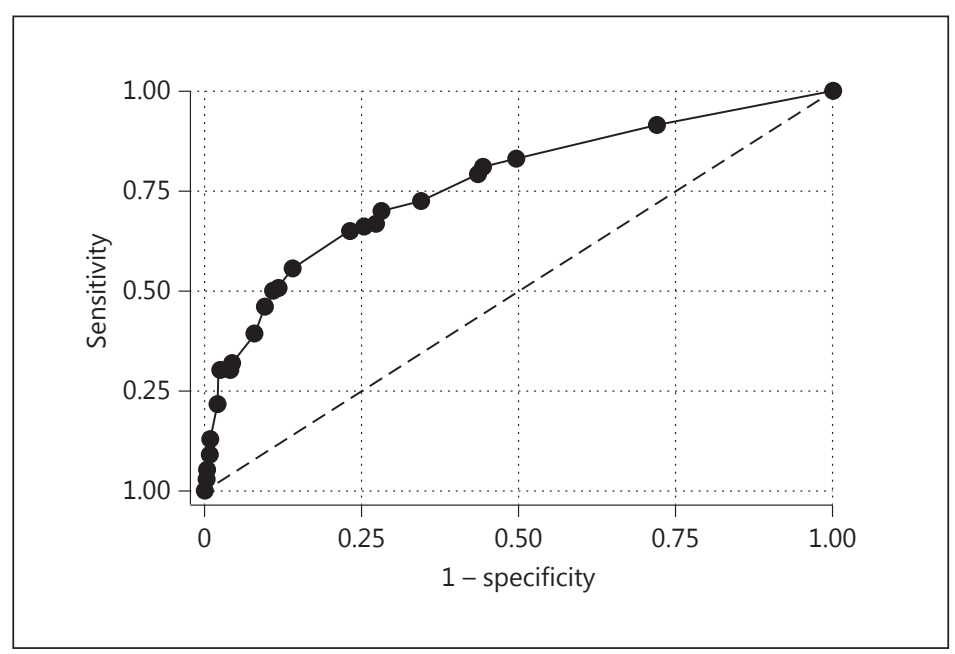

CIN after PCI is strongly associated with prolonged hospitalization, increased costs, increased rates of end-stage renal failure, myocardial infarction, repeat revascularization, and early and late mortality [3-5]. Patients who develop CIN and require dialysis after PCI have $40 \%$ in-hospital mortality and $80 \% 2$-year mortality rates [13].

The development of CIN is a sign of poor short- and long-term prognosis after STE-ACS and NSTE-ACS despite successful early coronary revascularization $[9,14]$. Preexisting renal impairment, diabetes, congestive heart failure, advanced age, anemia, use of high-contrast medium volume, and a reduced intravascular volume are risk factors for the development of CIN $[7,15,16]$.

The pathophysiologic mechanisms of CIN are complex, multifactorial, and incompletely understood. Possible mechanisms include intrarenal vasoconstriction, reduced renal blood flow, medullary hypoxia, oxidative stress, inflammation, endothelial dysfunction, and direct tubular epithelial cell injury by contrast medium [17]. CIN is a specific type of cardiorenal syndrome [18]. Renal injury starts with hemodynamic effects of myocardial infarction. Abnormal hemodynamic states lead to a decrease in renal blood flow, and the activation of immune and neurohormonal systems, such as the sympathetic nervous system and the reninangiotensin-aldosterone system, contributes to medullary hypoxia $[19,20]$. Direct cytotoxicity to renal tubular cells caused by the contrast agent then occurs. The results of the injury include renal vasoconstriction, impaired vasodilation, medullary hypoxia leading to oxidative stress, and tubular injury [21].

A recent study showed that a higher PLR value is related to the presence of coronary artery disease and is correlated with CRP and fibrinogen levels [22]. Both thrombocytosis and lymphocytopenia correlate with the degree of systemic inflammation, and PLR represents a novel marker incorporating both hematologic indices. We have hypothesized that PLR is not directly but possibly a result of the inflammatory process in CIN. In the present study, we demonstrate that a higher PLR value may be related to CIN.

In a previous study, the neutrophil-to-lymphocyte ratio has been shown to be associated with CIN [23]. With similar results obtained by PLR, we show that hematologic markers can be used in the diagnosis of CIN availability.

In our study, MVD, the presence of CTO in non-culprit vessels, high SYNTAX scores, and low stent diameters were associated with a higher incidence of CIN. In our opinion, high SYNTAX scores, MVD, and the presence of CTO in non-culprit vessels are related to more serious presentations (e.g., shock, arrhythmia), longer procedural duration, more contrast 
medium use, a higher incidence of no-reflow, and a lower LVEF. Thus, the renal vasoconstrictive response and the resulting corticomedullar hypoxia [20] may be aggravated in patients with high scores. It appears (although not statistically significant) that procedural success was considerably lower in the CIN group as compared to the no-CIN group. We think it may be due to the amount of contrast agent used and the total time of the procedure in the CIN group compared to the no-CIN group (although not statistically significant). The use of a greater contrast volume is associated with greater rates of CIN [24]. However, it was shown that the contrast medium volume does not have an effect on CIN if low-osmolar contrast agents and adequate hydration are used [25]. In our study, we did not find any effect of contrast agent volume on CIN. Two factors might be responsible for this result. First, we used low-osmolar agents and adequate hydration for our patients. Second, the amount of contrast volume used during the procedure was similar in our study population.

\section{Study Limitations}

This study has several limitations. First, we measured PLR only once at admission and without correction for potential variability in PLR levels. Second, the follow-up assessment of renal function in our study was performed 1-3 days after PCI; therefore, we might have missed a later increase in serum creatinine in some patients who did not have renal function deterioration within $72 \mathrm{~h}$ of their procedure. This might have resulted in a slight underestimation of CIN. Finally, we did not measure inflammatory indices except for hs-CRP.

\section{Conclusion}

Admission PLR levels may predict the development of CIN after PCI in patients with ACS. The results may be of clinical significance because PLR is easily available, widely used, and relatively cheap compared to most other laboratory markers.

\section{Disclosure Statement}

There are no conflicts of interest.

\section{References}

1 Goldfarb S, McCullough PA, McDermott J, et al: Contrast-induced acute kidney injury: specialty-specific protocols for interventional radiology, diagnostic computed tomography radiology, and interventional cardiology. Mayo Clin Proc 2009;84:170-179.

$\checkmark 2$ Finn WF: The clinical and renal consequences of contrast-induced nephropathy. Nephrol Dial Transplant 2006;21:i2-i10.

3 McCullough PA: Contrast-induced acute kidney injury. J Am Coll Cardiol 2008;51:1419-1428.

4 Recio-Mayoral A, Chaparro M, Prado B, et al: The reno-protective effect of hydration with sodium bicarbonate plus $\mathrm{N}$-acetylcysteine in patients undergoing emergency percutaneous coronary intervention: the RENO study. J Am Coll Cardiol 2007;49:1283-1288.

5 Lazaros G, Tsiachris D, Tousoulis D, et al: In-hospital worsening renal function is an independent predictor of one-year mortality in patients with acute myocardial infarction. Int J Cardiol 2012;155:97-101.

6 Bartholomew BA, Harjai KJ, Dukkipati S, et al: Impact of nephropathy after percutaneous coronary intervention and a method for risk stratification. Am J Cardiol 2004;93:1515-1519.

7 Mehran R, Aymong ED, Nikolsky E, et al: A simple risk score form prediction of contrast-induced nephropathy after percutaneous coronary intervention: development and initial validation. J Am Coll Cardiol 2004;44: 1393-1399.

8 Senoo T, Motohiro M, Kamihata H, et al: Contrast-induced nephropathy in patients undergoing emergency percutaneous coronary intervention for acute coronary syndrome. Am J Cardiol 2010;105:624-628. 


\section{CardioRenal Medicine}

Demircelik et al.: Association between Platelet-to-Lymphocyte Ratio and CIN in Patients Undergoing Percutaneous Coronary Intervention for ACS

-9 McCullough PA, Stacul F, Becker CR, et al; CIN Consensus Working Panel: Contrast-Induced Nephropathy (CIN) Consensus Working Panel: executive summary. Rev Cardiovasc Med 2006;7:177-197.

10 Lai Q, Lerut J: Reply to 'neutrophil and platelet-to-lymphocyte ratio: new predictors of dropout and recurrence after liver transplantation for hepatocellular cancer?' Transpl Int 2014;27:e34-e35.

11 Schiller NB, Shah PM, Crawford M, et al: Recommendations for quantitation of the left ventricle by two-dimensional echocardiography. American Society of Echocardiography Committee on Standards, Subcommittee on Quantitation of Two-Dimensional Echocardiograms (review). J Am Soc Echocardiogr 1989;2:358-367.

12 Azab B, Shah N, Akerman M, McGinn JT Jr: Value of platelet/lymphocyte ratio as a predictor of all-cause mortality after non-ST-elevation myocardial infarction. J Thromb Thrombolysis 2012;34:326-334.

13 McCullough PA, Soman SS: Contrast-induced nephropathy. Crit Care Clin 2005;21:261-280.

14 Fox CS, Muntner P, Chen AY, et al: Short-term outcomes of acute myocardial infarction in patients with acute kidney injury: a report from the national cardiovascular data registry. Circulation 2012;125:497-504.

15 McCullough PA, Wolyn R, Rocher LL, et al: Acute renal failure after coronary intervention: incidence, risk factors, and relationship to mortality. Am J Med 1997;103:368-375.

16 Wi J, Ko YG, Shin DH, et al: Prediction of contrast-induced nephropathy with persistent renal dysfunction and adverse longterm outcomes in patients with acute myocardial infarction using the Mehran risk score. Clin Cardiol 2013;36:46-53.

17 Goldenberg I, Matetzky S: Nephropathy induced by contrast media: pathogenesis, risk factors and preventive strategies. CMAJ 2005;172:1461-1471.

18 Ronco C, McCullough P, Anker SD, et al; Acute Dialysis Quality Initiative (ADQI) consensus group: Cardio-renal syndromes: report from the consensus conference of the acute dialysis quality initiative. Eur Heart J 2010;31: 703-711.

19 Ronco C, Haapio M, House AA, et al: Cardiorenal syndrome. J Am Coll Cardiol 2008;52:1527-1539.

-20 Heyman SN, Reichman J, Brezis M: Pathophysiology of radiocontrast nephropathy: a role for medullary hypoxia. Invest Radiol 1999;34:685-691.

21 McCullough PA: Acute kidney injury with iodinated contrast. Crit Care Med 2008;36(suppl 4):S204-S211.

22 Gary T, Pichler M, Belaj K, et al: Platelet-to-lymphocyte ratio: a novel marker for critical limb ischemia in peripheral arterial occlusive disease patients. PLoS One 2013;8:676-688.

-23 Kaya A, Kaya Y, Topçu S, Günaydin ZY, Kurt M, Tanboğa IH, Kalkan K, Aksakal E: Neutrophil-to-lymphocyte ratio predicts contrast-induced nephropathy in patients undergoing primary percutaneous coronary intervention. Angiology 2014;65:51-56.

-24 McCullough PA, Adam A, Becker CR, et al; CIN Consensus Working Panel: Risk prediction of contrast induced nephropathy. Am J Cardiol 2006;98:27K-36K.

25 Cho JY, Jeong MH, Hwan Park S, et al: Effect of contrast-induced nephropathy on cardiac outcomes after use of nonionic isosmolar contrast media during coronary procedure. J Cardiol 2010;56:300-306. 